\title{
CONOCIMIENTO ESPECIALIZADO DE LOS \\ ESTUDIANTES PARA MAESTRO: LA RESOLUCIÓN \\ DE UN PROBLEMA CON DIVISIÓN DE FRACCIONES
}

\author{
Ma Mar Liñán García, Víctor Barrera Castarnado y \\ José Manuel Infante Infante
}

\section{RESUMEN}

Analizamos los conocimientos, errores y estrategias de los estudiantes para maestro de primaria (EPM) que ponen en juego para resolver un problema aritmético elemental (PAE) de una etapa de estructura multiplicativa en el que se usa la división por fracción. En un paradigma interpretativo, se han tabulado las distintas acciones de los EPM ante este problema, tomando como marco de referencia el Conocimiento Especializado del Profesor, MTSK I. El análisis de los resultados confirma ciertas debilidades conceptuales y procedimentales en la división por fracción, aunque también hemos encontrado interpretaciones diferentes a las esperadas, pero correctas. Nuevas representaciones que podrían mostrar la posible especialización de su conocimiento.

Palabras clave: Conocimiento Especializado del Profesor de Matemáticas (MTSK), conocimiento de los temas matemáticos (KoT), conocimiento de la práctica matemática (KPM), conocimiento de la estructura matemática (KSM), formación inicial de profesores, división por fracción, Educación Primaria.

\section{TITLE: TEACHER TRAINEES' SPECIALIZED KNOWLEDGE: SOLVING A PROBLEM WITH DIVISION OF FRACTIONS}

\section{ABSTRACT}

We analyse the knowledge, errors and strategies that Prospective Primary Teachers (PPT) use in order to solve a one-step school arithmetic problems at a multiplying structural stage in which division through fractions is used. In an interpretative paradigm, the different actions of the PPT faced with this problem have been tabulated, taking as a reference framework the Mathematics Teachers' Specialised Knowledge, MTSK. An analysis of the results confirms that there are certain conceptual and procedural weaknesses in division through fractions, although we have found different interpretations to what we expected, but at the same time, correct. New representations which could demonstrate the possible specialisation of their knowledge.

Keywords: Mathematics Teacher's Specialized Knowledge (MTSK), Knowledge of Topics (KoT), Knowledge of the Practice of Mathematics (KPM), Knowledge of the Structure of Mathematics (KSM), Prospective teachers' training, divide by fraction, Primary Education

Correspondencia con los autores: Ma Liñán García <mlinan@ceuandalucia.es > Víctor Barrera Castarnado < vbarrera@ceuandalucia.es> José Manuel Infante Infante < jminfante@ceuandalucia.es >. Centro de Estudios Unversitario Cardenal Spínola CEU. Original recibido: 23-01-14. Original aceptado: 02-05-14 


\section{Planteamiento del problema}

Como profesores del área de Didáctica de la Matemática nos preocupa profundamente el facilitar que nuestros estudiantes se formen como maestros de matemáticas de calidad. Esta formación implica un análisis exhaustivo de todo el conocimiento que necesita un buen profesor de matemáticas.

Como recoge $\mathrm{Ma}$ (1999) el conocimiento profundo de la matemática fundamental es imprescindible para la buena labor de un maestro. Evidentemente, esto no es suficiente: en las últimas décadas ha habido cambios en lo que se considera el conocimiento profesional de un docente de matemáticas, intentando integrar el conocimiento disciplinar y el derivado de la investigación didáctica (Ball, Thames, Phelps, 2008; Shulman, 1986). Considerando que todo el conocimiento matemático de un docente ha de ser especializado (Carrillo, Climent, Contreras y MuñozCatalán, 2013), seguimos el modelo Conocimiento Especializado del Profesor de Matemáticas (MTSK) propuesto por el Seminario de Investigación en Didáctica de la Matemática de la Universidad de Huelva (SIDM). En este modelo se consideran como conocimiento específico de un profesor de matemáticas el conocimiento matemático en sí (conocimiento de los temas, de la estructura de la matemática y de la práctica matemática) y el conocimiento pedagógico del contenido (conocimiento de la enseñanza de las matemáticas, del aprendizaje de las mismas y de los estándares de aprendizaje), además de las concepciones sobre las matemáticas y su enseñanza y aprendizaje.

En este trabajo nos planteamos analizar el conocimiento matemático que tienen los estudiantes para maestro (EPM) referido a la resolución de un problema multiplicativo en el que interviene la división de fracciones. Para fijar ese contenido matemático, hemos considerado tanto los documentos oficiales en los que se desarrolla el Currículo de matemáticas español, como otros documentos internacionales (por ejemplo Principles and Standards for School Mathematics, NCTM 2000), en los que se da mucha importancia a la resolución de problemas: "Los procesos de resolución de problemas constituyen uno de los ejes principales de la actividad matemática y deben ser fuente y soporte principal del aprendizaje matemático a lo largo de la etapa" (Real Decreto I5 I3/2006, p. 43096). La resolución de problemas se considera, por tanto, como instrumento para generar conocimiento con significado. Por otro lado, en la literatura de investigación se ha mostrado la dificultad que tienen sujetos de diferentes niveles académicos para darle sentido a la división de fracciones (Ma, 1999, Contreras, 2013). Márquez (2013), citando a su vez a Flores (2002), Flores, 
Turner y Bachea (2005), Lo y Luo (20I2), Nillas (2003), Perlwitz (2004, 2005), Peck y Wood (2008), Gregg y Gregg (2007), advierte de la necesidad de que los profesores posean un conocimiento especializado para la enseñanza de la división de fracciones.

Si nos centramos en PAEs ${ }^{2}$ de una etapa, multiplicativos asimétricos de división, los papeles que juegan cada una de las cantidades involucradas en el enunciado son: dividendo, es la cantidad a partir en partes iguales; divisor y cociente tienen significados intercambiables, uno de ellos indica la cantidad de partes equitativas en las que se quiere dividir la cantidad total, y el otro representa la cantidad relativa a cada una de las partes. Los modelos semánticos asociados a cada caso son: si el divisor representa el número de partes de igual cantidad en las que se quiere partir el dividendo, se conoce con el nombre de división-reparto o división partitiva. En el otro caso se llama división-medida o división cuotición.

En la división partitiva, el divisor indica el número de partes iguales en que se parte la cantidad total, y el cociente un número homogéneo al dividendo. Esta interpretación no tiene sentido cuando el divisor es una fracción. ¿Qué significado puede tener partir $2 / 5$ en 3/8 partes? Con esto tenemos que en la división partitiva no hay continuidad semántica cuando el divisor es una fracción. Sin embargo, en la división cuotitiva el divisor es un número homogéneo al dividendo, que indica el número de "ítems" que corresponde a cada parte. Esta interpretación no tiene sentido cuando el divisor es mayor al dividendo. Si bien, en este caso la discontinuidad semántica se puede rehuir, por ejemplo en los casos de medida, utilizando subunidades.

La división de fracciones está considerada como operación de algoritmo fácil pero de compleja comprensión. Se necesita un aprendizaje significativo, con comprensión, que permita a los estudiantes su aplicación en los contextos y situaciones que la requieran. Es un algoritmo fácil de mecanizar, pues consiste en un procedimiento básico relacionado con los productos cruzados de los elementos de los dos miembros de la división o con el producto por la fracción inversa; sin embargo su aplicación en la resolución de problemas contextualizados plantea dificultad al no comprender las relaciones que se establecen entre las fracciones que intervienen en la situación a resolver. De hecho, $\mathrm{Ma}$ (1999) describe en su trabajo la dificultad que mostraron los profesores estadounidenses estudiados en plantear un problema que se resolviera con la división por fracción pues, a pesar de que conocían el algoritmo, en su mayoría planteaban una representación de multiplicación por fracción.

Son muchas las investigaciones que se centran en determinar qué aspectos influyen en la selección de la operación elegida para resolver un problema multiplicativo. Entre 
otras destacamos una mala generalización de algoritmos con números naturales, equívocos entre el concepto que tienen de fracción y el de problema multiplicativo, confusión entre el algoritmo y el significado de los conceptos en juego, los modelos de situación utilizados por los profesores para resolver los problemas, y el tipo y naturaleza de los números implicados en el problema (Gómez, 2013).

En cualquier caso la discontinuidad semántica de la división de fracciones afecta a la hora de identificar qué operación resuelve el problema, pues no es fácil encontrar la "palabra clave" que ayude en este sentido, además de tener dificultad en diferenciar entre dividendo y divisor, cuestión que en PAEs con número naturales es más fácil.

Nuestro objetivo es analizar qué conocimiento especializado tienen los EPM, que ya han recibido la formación correspondiente a las matemáticas específicas para maestros (en nuestro caso, estudiantes de $2^{\circ}$ de grado de educación primaria), sobre la división de entero entre fracción, por lo que nos planteamos la siguiente pregunta de investigación: ¿Qué conocimiento especializado de los EPM subyace en el uso de heurísticos para la resolución de un problema con división de entero entre fracción?

\section{Marco teórico}

\section{I. Conocimiento especializado}

Petrou y Goulding (20I I), en su recorrido sobre el conocimiento del profesor desde finales de los 80 hasta la actualidad, declaran que Shulman (1986) y sus posteriores variantes incorporaron un proceso crucial de transformación fijado en la interpretación y la representación de conceptos de las materias y en su ajuste a las situaciones escolares generales y su adaptación a grupos particulares de estudiantes, lo que ha llamado la atención de numerosos investigadores. Fue su trabajo con profesores de secundaria lo que permitió a Shulman (op. cit.) desarrollar un marco teórico sobre el conocimiento del profesor que incluía por primera vez, entre otros, el conocimiento del contenido y el conocimiento de la didáctica específica. A pesar de no estar referido exclusivamente a las matemáticas, este marco se acercaba más a la realidad de ese conocimiento del profesor, pero investigaciones posteriores (Ball, Thames y Phelps, 2008), han argumentado que la clasificación del conocimiento propuesto por Shulman no siempre está clara, además de no admitir interacciones entre categorías y de ignorar la naturaleza dinámica del conocimiento de la enseñanza. 
Posteriormente, Fennema y Franke (1992), centrados ya en la enseñanza de las matemáticas, asumen ese dinamismo y añaden las concepciones del profesor como elemento determinante en su práctica. No fue hasta Ball et al. (op. cit.) que se examinaron las ideas de Shulman y se diferenció claramente entre el conocimiento del contenido y el conocimiento didáctico del contenido, aunque se vuelve a perder la importancia que Fennema y Franke (op. cit.) le atribuyen a las concepciones del profesor.

Godino (2009) apunta que estos modelos incluyen categorías demasiado generales y propone la utilidad de construir otros que consideren los conocimientos puestos en juego en una "enseñanza efectiva" (op. cit., p. 19), que tenga en cuenta la competencia, eficacia y la idoneidad de la enseñanza. Así, la Competencia Matemática para la Enseñanza, MPT ${ }^{3}$ (Conner, Wilson y Jung Kim, 20II) que considera la competencia en el trabajo matemático para la enseñanza, en la actividad matemática y en el promover metas matemáticas, pretende, partiendo del modelo Mathematics Knowledge for Teaching (MKT) propuesto por Ball y colaboradores (2008), generar un marco más amplio y dinámico para analizar el conocimiento matemático: por primera vez observamos la consideración de que todo el conocimiento matemático del profesor ha de ser especializado. En esta misma línea, el subdominio correspondiente al Conocimiento Especializado (Ball et al., op. cit.) está siendo objeto de discusión por los investigadores, que consideran genera problemas de delimitación con el subdominio del Conocimiento Común (Flores, Escudero y Carrillo, 20I3).

Con estos antecedentes, el grupo SIDM presenta un modelo analítico, actualmente en desarrollo, basado en que todo el conocimiento del profesor de matemáticas es especializado porque deriva de su profesión (Carrillo, Climent, Contreras, Muñoz-Catalán, op. cit.), luego no puede ser específica solo una parte de dicho conocimiento en tanto que este es específico para enseñar matemáticas (Liñán y Contreras, 2013). Este nuevo modelo, uno de los pilares en el que vamos a apoyar nuestro trabajo, se ha denominado Conocimiento Especializado del Profesor de Matemáticas (MTSK).

Podemos ver el hexágono de la Figura I dividido en dos grandes partes: el Conocimiento Matemático (MK) y el Conocimiento Didáctico del contenido $(P C K)$, unidas en el centro por las concepciones sobre la matemática y sobre la enseñanza y el aprendizaje de la misma (Carrillo et al., op. cit.; Montes, Aguilar, Carrillo; Muñoz-Catalán, 2013). 


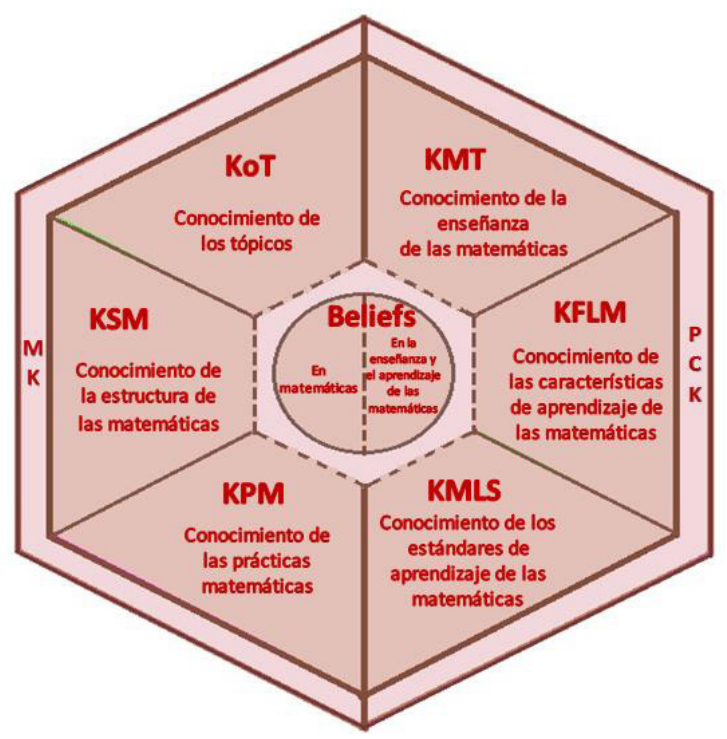

Figura I. MTSK

El conocimiento matemático, donde nos vamos a centrar para nuestro estudio, está subdividido en tres subdominios:

- Conocimiento de los Temas Matemáticos (KoT), que incluye la fundamentación matemática y propiedades, los procedimientos asociados y las diferentes formas de representación, los contextos en los que situar un mismo concepto (fenomenología) y los diferentes significados que se pueden atribuir a un contenido (definiciones); podríamos incluir aquí también el conjunto de ejemplos que un maestro plantearía para trabajar los temas considerados desde distintos puntos de vista.

- Conocimiento de la Estructura Matemática (KSM), entiende que los conceptos no son aislados, sino que están integrados en un sistema de conexiones. Incluye los elementos tanto más avanzados como más elementales de tal objeto, lo que permite trabajar la matemática avanzada desde un punto de vista elemental (Conexiones de Simplificación) y viceversa (Conexiones de Complejización). Completan este subdominio las Conexiones Transversales, entre contenidos con una cualidad común que les relaciona, y las Conexiones Auxiliares, aquellas que sirven como herramienta para obtener otros resultados. 
- Conocimiento de la Práctica Matemática (KPM), donde trabajaríamos las formas de hacer y proceder, incluso pensar, en matemáticas que un maestro tiene que conocer, como son las diferentes formas de demostrar, el significado de definición, axioma o teorema como elementos constituyentes de la matemática, o el conocimiento de la sintaxis matemática. Trataríamos aquí los heurísticos puestos en juego en la resolución de un problema.

El conocimiento didáctico del contenido, que, aunque no forma parte de este estudio, consideramos enriquecedor mostrar, contiene tres nuevos subdominios:

- Conocimiento de la Enseñanza de las Matemáticas (KMT), estrategias de enseñanza, recursos y materiales que el maestro debe conocer, incluyendo aquí aquello que permita ayudar a los alumnos a construir conceptos matemáticos y que consiga despertar su intuición; así mismo, todas aquellas habilidades del maestro para habilitar al alumnado a superar sus propios errores.

- Conocimiento de las características de aprendizaje matemático (KFLM), el saber cómo aprenden los alumnos el contenido matemático y el conocimiento de las características de ese proceso de comprensión, así como de los errores, dificultades, y obstáculos asociados a cada concepto, ideas previas de los alumnos y lenguaje que usan.

- Conocimiento de los estándares de aprendizaje (KMLS), que además del conocimiento del currículo oficial, incluye el conocimiento de las producciones de las distintas investigaciones en el área de didáctica de las matemáticas respecto a lo esperado en el aprendizaje de cada etapa, es decir el conocimiento de lo que el estudiante puede alcanzar, lo que se espera, así como la secuenciación de dicho conocimiento.

Dado que nuestro objetivo es aproximarnos al conocimiento matemático especializado de un EPM puesto en juego en la resolución de un problema multiplicativo, utilizaremos el modelo MTSK para determinarlo y analizarlo. Debemos aclarar que, a pesar de que el modelo analítico MTSK estudia la práctica del maestro, podemos asumirlo como referente de los componentes deseables en el conocimiento especializado de un maestro y como consecuencia, como una primera aproximación a lo que un EPM debería conocer para su futura práctica. Solo analizaremos el contenido matemático, pues en la forma en la que se les ha planteado el problema y su resolución, no cabe la parte correspondiente a la enseñanza y el aprendizaje; mostramos a continuación los indicadores, por categoría dentro de cada subdominio, que vamos a tener en cuenta dentro del MK. 


\begin{tabular}{|c|c|c|}
\hline \multicolumn{3}{|c|}{ КoT } \\
\hline \multirow{3}{*}{ Fenomenología } & Interpretación cuotitiva de fracción. & $\begin{array}{l}\text { Saber que la fracción que está involucrada en } \\
\text { el problema indica medida. }\end{array}$ \\
\hline & Medida y magnitud. & $\begin{array}{l}\text { Saber qué es la magnitud peso y las unidades } \\
\text { en las que se mide. Saber que es necesario } \\
\text { tener todas las cantidades en juego en la } \\
\text { misma unidad. }\end{array}$ \\
\hline & Proporcionalidad directa. & $\begin{array}{l}\text { Saber identificar situaciones en las que se } \\
\text { establezca una relación de proporcionalidad } \\
\text { directa. }\end{array}$ \\
\hline Propiedades & $\begin{array}{l}\text { Propiedades de los números } \\
\text { racionales respecto de la suma y la } \\
\text { multiplicación. }\end{array}$ & $\begin{array}{l}\text { Saber aplicar las propiedades conmutativa, } \\
\text { asociativa, distributiva, elemento neutro, } \\
\text { elemento opuesto y elemento inverso. }\end{array}$ \\
\hline \multirow{2}{*}{$\begin{array}{l}\text { Registros de } \\
\text { representación }\end{array}$} & Números decimales. & $\begin{array}{l}\text { Saber que una fracción es una } \\
\text { representación de un número decimal. }\end{array}$ \\
\hline & $\begin{array}{l}\text { Representación gráfica de } \\
\text { fracciones. }\end{array}$ & $\begin{array}{l}\text { Saber utilizar las representaciones discreta y } \\
\text { continua de una fracción. }\end{array}$ \\
\hline Definiciones & División entre fracción. & $\begin{array}{l}\text { Saber qué significa dividir cualquier cantidad } \\
\text { entre una fracción (Ma, 1999). }\end{array}$ \\
\hline \multirow{3}{*}{ Procedimientos } & $\begin{array}{l}\text { Algoritmo división de entero entre } \\
\text { fracción }\end{array}$ & $\begin{array}{l}\text { Conocer el procedimiento de división de } \\
\text { fracciones. }\end{array}$ \\
\hline & $\begin{array}{l}\text { Representaciones de un número } \\
\text { racional. }\end{array}$ & $\begin{array}{l}\text { Conocer las transformaciones entre fracción } \\
\text { y expresión decimal o viceversa. }\end{array}$ \\
\hline & Medida. & $\begin{array}{l}\text { Utilizar los divisores de una unidad para } \\
\text { evitar representaciones decimales. }\end{array}$ \\
\hline \multicolumn{3}{|c|}{ KSM } \\
\hline $\begin{array}{l}\text { Conexión de } \\
\text { complejización }\end{array}$ & $\begin{array}{l}\text { Relación entre la interpretación } \\
\text { cuotitiva de una fracción y la medida. }\end{array}$ & $\begin{array}{l}\text { Saber interpretar la fracción como cuántas } \\
\text { veces está contenido un número en otro. }\end{array}$ \\
\hline $\begin{array}{l}\text { Conexión de } \\
\text { contenidos } \\
\text { transversales } \\
\end{array}$ & $\begin{array}{l}\text { Relación entre la división entre } \\
\text { fracciones y la medida }\end{array}$ & $\begin{array}{l}\text { Saber interpretar la división como una } \\
\text { comparación en el sentido amplio de la medida. }\end{array}$ \\
\hline $\begin{array}{l}\text { Conexión de } \\
\text { simplificación }\end{array}$ & $\begin{array}{l}\text { Relación entre la división de } \\
\text { fracciones y la resta reiterada. }\end{array}$ & $\begin{array}{l}\text { Saber que una interpretación de la división } \\
\text { es equivalente a una resta reiterada. }\end{array}$ \\
\hline $\begin{array}{l}\text { Conexión } \\
\text { auxiliar }\end{array}$ & $\begin{array}{l}\text { Relación entre las propiedades } \\
\text { de la suma y multiplicación de los } \\
\text { números racionales. }\end{array}$ & $\begin{array}{l}\text { Saber aplicar las citadas propiedades resolver } \\
\text { una ecuación lineal. }\end{array}$ \\
\hline \multicolumn{3}{|c|}{ KPM } \\
\hline $\begin{array}{l}\text { Formas de } \\
\text { proceder }\end{array}$ & $\begin{array}{l}\text { Heurísticos aplicables a la resolución } \\
\text { de problemas. }\end{array}$ & $\begin{array}{l}\text { Conocer algunos de los heurísticos para } \\
\text { cada una de las fases de la resolución de } \\
\text { problemas }\end{array}$ \\
\hline
\end{tabular}

Tabla I. Indicadores del Conocimiento Matemático Especializado a analizar en el estudio 


\subsection{Resolución de problemas}

Por otro lado, y puesto que se trata al fin de analizar no solo el conocimiento matemático sino también la estrategia de resolución del problema multiplicativo en sí, debemos tener en cuenta también las fases en la resolución de problemas propuesta por Carrillo (1996), apoyada a su vez en autores como Polya (1995), Kantowski (198I), Schoenfeld (1994). Carrillo (op. cit.) considera que se debe situar el problema en la aplicación del conocimiento matemático, especializado en este caso, a escenarios desconocidos o no familiares.

Para el estudio de nuestros casos, interpretaremos la resolución de problemas como un proceso, ya que pretendemos observar los razonamientos de los estudiantes en cada uno de los pasos que dan para su resolución. Además, queremos analizar qué fases de las descritas por Schoenfeld (op. cit.), apoyadas en Polya (op. cit.) y descritas en profundidad por Echenique (2006), forman parte de los distintos procesos que eligen los EPM para su resolución:

I. Comprensión del problema: lectura y análisis. Tanto en lo referente a la comprensión lectora como al interés por resolverlo, precisa del análisis del texto para resaltar los contenidos clave que les permitan representarlo. Los posibles errores que nos podremos encontrar estarán relacionados con la falta de comprensión lectora o el bloqueo ante una situación desconocida. Heurísticos: organizar la información, ejemplificar, expresar en otros términos.

2. Concepción de un plan: exploración y planificación, analizando las relaciones entre los datos conocidos y desconocidos, elección de una estrategia adecuada. Los errores asociados estarán relacionados con la falta de herramientas, poca experiencia en la resolución de problemas o un conocimiento matemático especializado mal asimilado. Los heurísticos relacionados serían: simplificar, estimar, buscar regularidades para la posible generalización, razonamiento por contradicción, jerarquizar, descomponer el problema, conjeturar.

3. Ejecución del plan, estimando el posible resultado, teniendo en cuenta las magnitudes, para aplicar finalmente los procedimientos diseñados en el paso anterior. Los posibles errores se derivarán de la falta de dominio en técnicas y procedimientos de cálculo, manejo de magnitudes y unidades. Heurísticos: actuar con orden y precisión, explicar resultado de la ejecución, registrar los cálculos y resaltar los logros intermedios. 
4. Visión retrospectiva, en la que se debe comparar el resultado con la estimación y el enunciado del problema, verificando los pasos del razonamiento seguido $y$, finalmente, pensar en otras posibles formas de resolución. Los errores suelen estar asociados a una falta de espíritu crítico. Heurísticos: analizar la consistencia del proceso y de la solución, expresarla de otro modo, generalizar.

A continuación describimos los heurísticos ${ }^{5}$ que Carrillo (1996) propone en cada una de las fases - mantenemos la nomenclatura propuesta por Polya (1995)-:

\begin{tabular}{c|l|l}
\hline Fase & \multicolumn{1}{|c|}{ Heurístico } & \multicolumn{1}{c}{ Indicador } \\
\hline \multirow{4}{*}{ Comprensión } & \multirow{2}{*}{ Organizar la información } & Imagina mentalmente la situación \\
\cline { 2 - 3 } & & Utiliza algún esquema gráfico \\
\cline { 2 - 3 } & \multirow{2}{*}{ Expresar en otros términos } & Formula con otras palabras el enunciado \\
\cline { 3 - 3 } & & Introduce notación matemática \\
\hline
\end{tabular}

\begin{tabular}{l|l|l}
\hline \multirow{4}{*}{$\begin{array}{l}\text { Planificación y y } \\
\text { Exploración }\end{array}$} & Estimar & $\begin{array}{l}\text { Tiene una idea de cuánto debe valer la } \\
\text { solución }\end{array}$ \\
\cline { 2 - 3 } & Tantear & $\begin{array}{l}\text { Prueba con cantidades más pequeñas para } \\
\text { obtener un modelo }\end{array}$ \\
\cline { 2 - 3 } & Descomponer el problema & $\begin{array}{l}\text { Divide datos en partes congruentes para } \\
\text { reiterar una operación }\end{array}$ \\
\hline
\end{tabular}

\begin{tabular}{l|l|l}
\hline \multirow{4}{*}{ Ejecución } & Registrar todos los cálculos & $\begin{array}{l}\text { Anota de manera ordenada las operaciones } \\
\text { que piensa le pueden llevar a la solución }\end{array}$ \\
\cline { 2 - 3 } & Actuar con orden & $\begin{array}{l}\text { Organiza el proceso de resolución de } \\
\text { problema de manera coherente }\end{array}$ \\
\cline { 2 - 3 } & Explicar el estado de la ejecución & $\begin{array}{l}\text { Justifica los pasos dados en el proceso de } \\
\text { resolución }\end{array}$ \\
\hline
\end{tabular}

\begin{tabular}{|c|c|c|}
\hline \multirow{4}{*}{ Verificación } & $\begin{array}{l}\text { Analizar la consistencia de la } \\
\text { solución }\end{array}$ & Comprueba si la solución tiene sentido \\
\hline & Expresar la solución de otra forma & Simplifica del resultado \\
\hline & Analizar la consistencia del proceso & Justifica el procedimiento y el resultado \\
\hline & $\begin{array}{l}\text { Analizar si se puede llegar al } \\
\text { resultado de otra manera }\end{array}$ & $\begin{array}{l}\text { Busca otros procedimientos para llegar al } \\
\text { resultado }\end{array}$ \\
\hline
\end{tabular}

Tabla 2. Heurísticos según las fases de resolución de un problema 


\section{Metodología}

Para responder a nuestra pregunta de investigación ¿Qué conocimiento especializado de los EPM subyace en el uso de heurísticos para la resolución de un problema con división de fracciones? nos posicionamos en un paradigma interpretativo: queremos observar e interpretar una realidad en su entorno, ya que conocer no consiste en la interiorización de una copia de la realidad exterior, sino que implica una interacción con el objeto del conocimiento, a través del cual el sujeto interpreta y reconstruye los significados puestos en juego (Muñoz-Catalán, 20I2).

Nuestro diseño de investigación consiste en el estudio de casos partiendo de una tabulación que nos ha permitido agrupar las distintas acciones de los EPM ante el problema planteado, lo que nos servirá para seleccionar los casos más significativos y hacer con ellos un estudio más profundo.

Determinado el marco de referencia para este estudio, MTSK, ya descrito en el apartado anterior, la herramienta de recogida de datos ha consistido en el planteamiento del siguiente problema multiplicativo de una etapa, en el que se presenta la división de entero entre fracción:

Ana tiene un saco con $55 \mathrm{Kg}$ de garbanzos y los quiere repartir en paquetes de $3 / 4$ de Kg. ¿Cuántos paquetes obtendrá? ¿Cuántos kilos de garbanzos le sobrarán?

Se solicitó a los alumnos que, además de resolver la situación planteada, plasmaran las etapas en la resolución de la misma y todos los razonamientos que les pudieron llevar a las conclusiones finales.

Los participantes han sido en total I 16 EPM de $2^{\circ}$ curso de Grado en Educación Primaria, de los que 40 pertenecen a la Facultad de Educación de la Universidad de Sevilla y el resto al Centro de Estudios Universitarios Cardenal Spínola CEU6; todos ellos han recibido la formación en el contenido matemático específico y están cursando, en el momento de la toma de datos para esta investigación, la correspondiente al contenido didáctico.

\section{Análisis de la información}

Mostramos a continuación el análisis de las resoluciones al problema planteado y descrito en el apartado Metodología.

Basándonos en las tablas 1 y 2 presentadas en el marco teórico, referidas a los indicadores del MTSK y a los heurísticos propuestos por Carrillo (1996), presentamos el siguiente análisis de los resultados. 


\section{I. Conocimiento de los Temas Matemáticos (KoT).}

- Fenomenología: Interpretación cuotitiva de la división.

Tan solo tres de los EPM analizados razonan que de cada kilogramo se obtiene un paquete de $3 / 4 \mathrm{Kg}$ sobrando $1 / 4 \mathrm{Kg}$; con los "cuartos" sobrantes de los $55 \mathrm{Kg}$, vuelven a realizar el mismo razonamiento considerando lo finalmente sobrante.

Cuarenta y dos EPM realizan una división de $55 \mathrm{Kg}$ entre $3 / 4 \mathrm{Kg}$, sabiendo interpretar que el cociente indica el número de paquetes de $3 / 4 \mathrm{Kg}$ y el resto los kilos de garbanzos que quedan sin empaquetar. Sin embargo, veintitrés EPM realizan la misma división, pero no son capaces de interpretar el significado del resto. Por otro lado, trece EPM utilizan también correctamente el citado procedimiento, pero muestran dificultades al interpretar ambos, el cociente y resto obtenidos.

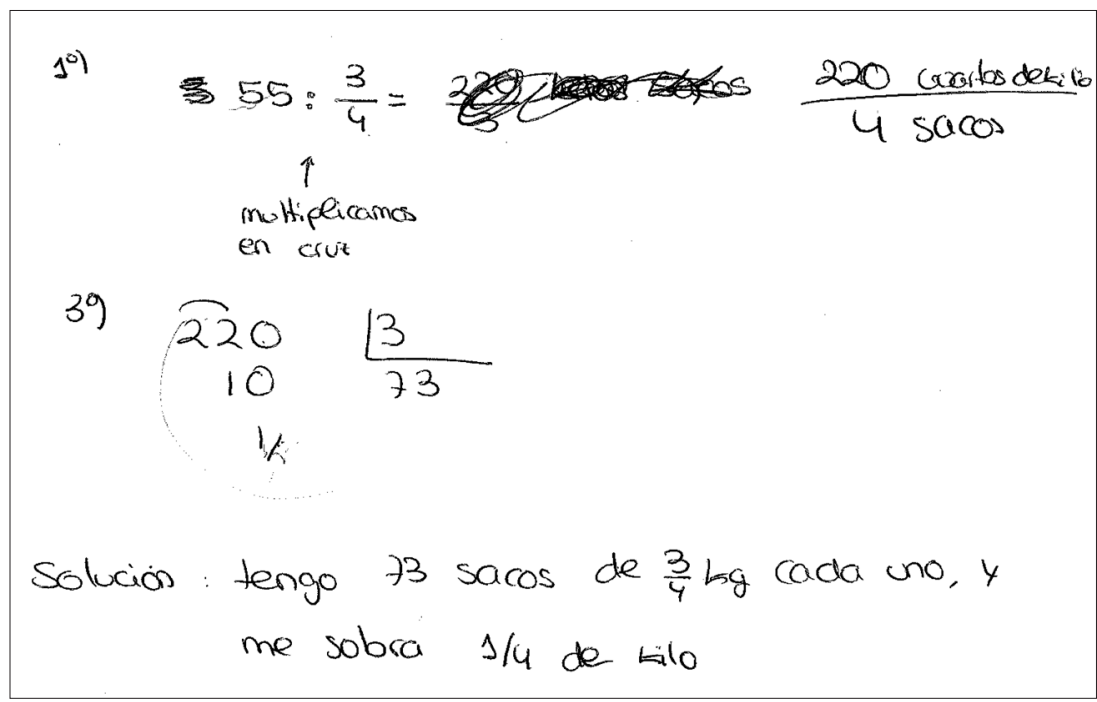

Ilustración I. Interpretación correcta de la división por fracción

Dos estudiantes que, fijándose que 54 es divisible entre 3 , realizan el reparto de esos $54 \mathrm{Kg}$ entre $3 / 4$, obteniendo así una cantidad exacta de paquetes y dándose cuenta de que, del $\mathrm{Kg}$ restante, pueden sacar un paquete más, concluyendo que les sobra tan solo $1 / 4 \mathrm{Kg}$. 
- Fenomenología: Medida y magnitud.

Once estudiantes utilizan un algoritmo correcto para resolver el problema, pero no indican ninguna de las unidades de medida involucradas en él, por lo que el significado del problema y su solución quedan descontextualizados.

Siete estudiantes, por otro lado, no saben interpretar correctamente la "división de unidades": al dividir kilogramos entre kilogramos por paquete, obtenemos paquetes.

- Fenomenología: Proporcionalidad directa.

Cuatro EPM interpretan erróneamente la situación como proporción directa: si de I $\mathrm{Kg}$ sale un paquete de $3 / 4 \mathrm{Kg}$, de $55 \mathrm{Kg}$ saldrán 55 paquetes - obviando los paquetes que se forman con cada cuarto sobrante - en lugar de hacer la proporción con $3 / 4$, lo que sí generaría una razón correcta.

Catorce estudiantes utilizan la regla de tres para conseguir interpretar el sobrante de garbanzos. En este caso utilizan correctamente el algoritmo de la división de entero entre fracción, pero al no ser una división exacta sacan cifras decimales, sin considerar que el número de paquetes debe ser entero. Para darle significado al resultado utilizan la regla de tres, teniendo en cuenta que si con $55 \mathrm{~kg}$ obtienen 73 ' 33 paquetes, los 0 ' 33 paquetes corresponderán con $0.25 \mathrm{~kg}$.

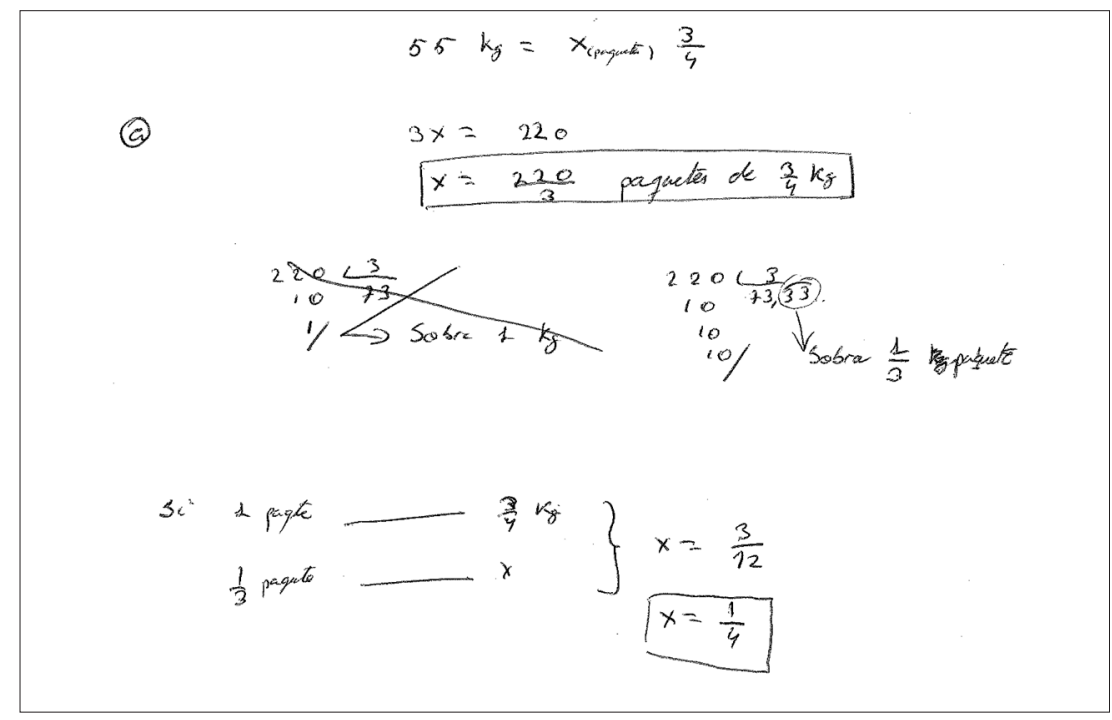

Ilustración 2. Interpretación proporcional del problema. 
- Propiedades: Propiedades de los números racionales respecto de la suma y la multiplicación

No existe referencia al uso de estas propiedades en el problema propuesto.

- Registros de representación: Números decimales.

Veintiún estudiantes cambian la expresión fraccionaria a decimal para evitar así la división por fracción, realizando algunos de ellos posteriormente una conversión de las unidades de la magnitud peso para trabajar con números enteros

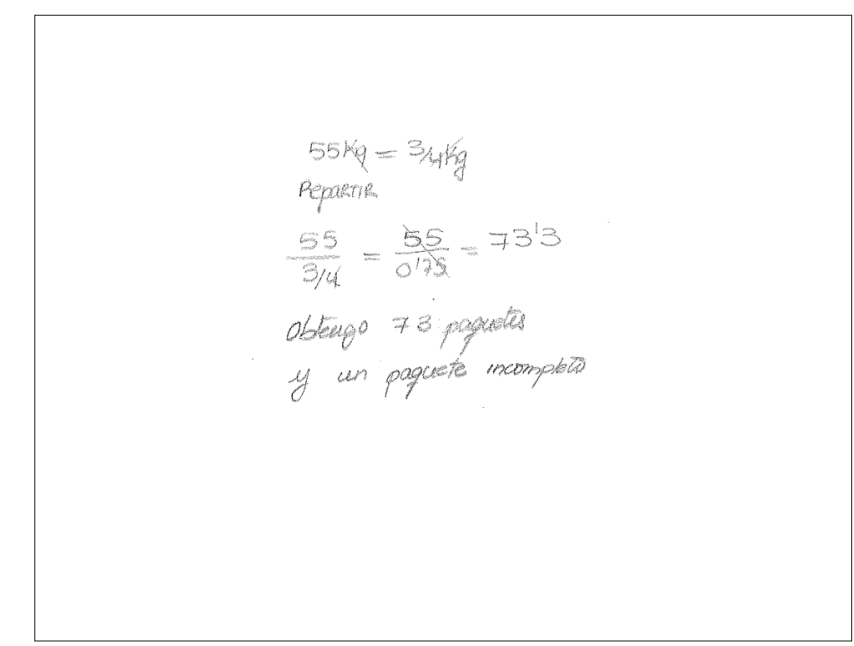

Ilustración 3. Paso de fracción a decimal para evitar división por fracción

- $\quad$ Registros de representación: Representación gráfica de fracciones

Aunque sean pocos, destacamos la representación elegida por dos EPM, que podríamos llamar "mixta", pues simbolizan los kilos de forma discreta y cada kilo como un todo continuo dividido en cuatro partes congruentes de las que toman tres, reagrupando posteriormente los cuartos sobrantes en nuevos paquetes de 3/4. Sin embargo, la mayoría no utiliza representación gráfica alguna y unos pocos usan figuras que no aportan nada a la resolución del problema. 


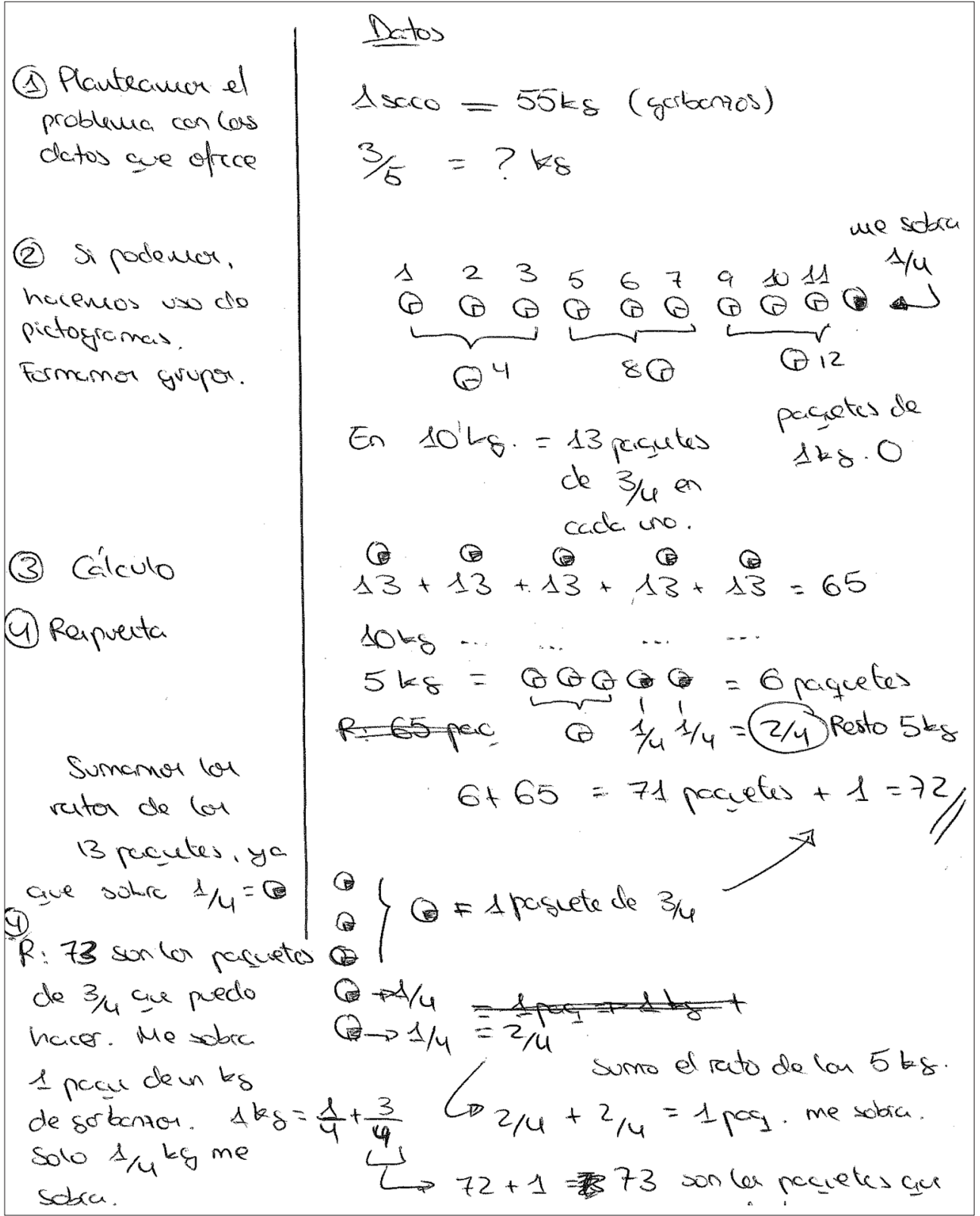

Ilustración 4. Representación gráfica "mixta" 
- Definiciones: División entre fracción

No se aprecia una referencia clara al significado de la división por fracción, a pesar de que es el contexto del problema, pues 74 estudiantes no han identificado la representación propuesta, bien por no haberle dado un significado al algoritmo o bien por no haberlo planteado correctamente.

- Procedimientos: Algoritmo división de entero entre fracción

Dieciséis EPM procesan correctamente el algoritmo de la división de entero entre fracción, interpretando bien los resultados obtenidos como cociente y resto. Sin embargo, dado que no tenemos constancia del argumento que les ha llevado a utilizar este procedimiento, hemos considerado que no debíamos ubicar este resultado en la interpretación cuotitiva de la división.

Finalmente, tenemos 34 EPM que realizan de modo incorrecto el algoritmo, decidiendo que el resultado se deriva de la multiplicación de 3/4 por 55 .

- Procedimientos: Representaciones de un número racional

Anteriormente hemos mencionado que más de veinte estudiantes cambian la expresión fraccionaria a decimal para evitar así la división por fracción, dejando patente el conocimiento asociado a las distintas representaciones de un número racional.

- Procedimientos: Medida

De la misma forma que en el párrafo anterior, primero generan una representación decimal del número, realizando algunos de ellos posteriormente una conversión correcta de las unidades de la magnitud peso evitando el trabajo con decimales, lo que denota el conocimiento asociado.

\subsection{Conocimiento de la estructura matemática (KSM)}

- Conexión de complejización: Relación entre la interpretación cuotitiva de la fracción y la medida.

Los dos EPM que han realizado una representación "mixta" (ver Registros: Representación gráfica de fracciones) muestran una interpretación de la fracción como medida al representar tres de las cuatro partes congruentes en las que queda dividido cada kilogramo del conjunto total. 
Otros dos EPM, sin embargo, interpretan como $3 / 4$ del total de garbanzos lo que en realidad se plantea en el problema como $3 / 4$ de kilogramo.

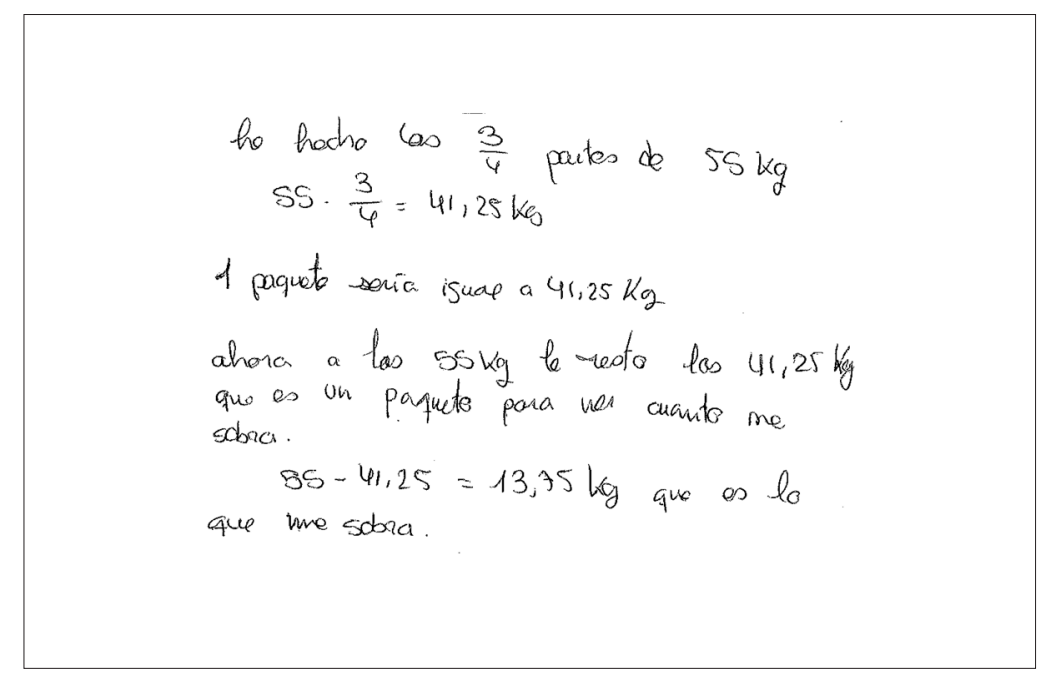

Ilustración 5. Confusión entre 3/4 Kg y 3/4 del total

- Conexión de contenidos transversales: Relación entre la división entre fracciones y la medida

Cuarenta y dos EPM interpretan correctamente el significado de la división como la comparación en el sentido amplio de la medida, pues muestran que al dividir los kilos totales entre $3 / 4$ obtienen el número de veces que "cabe" $3 / 4$ en 55 , es decir, el número de paquetes de $3 / 4 \mathrm{Kg}$ y, el resto de la división, lo que queda sin agrupar.

- Conexión de simplificación: Relación entre la división entre fracciones y la resta reiterada

Solo un EPM interpreta que la resta reiterada de $3 / 4$ de kilo a los 55 que componen el total equivale a la división, razonando que cada cuatro veces que resta los $3 / 4$, habrá reducido la cantidad inicial en 3 kilos. Consecuentemente, si divide el total de garbanzos por tres obtiene agrupaciones de 4 paquetes de $3 / 4$, lo que finalmente le lleva a que debe multiplicar por cuatro para obtener el total de paquetes pedidos. Finalmente, interpreta el resto de esa división como un kilo sobrante, del cual puede obtener otro paquete más de $3 / 4$. 


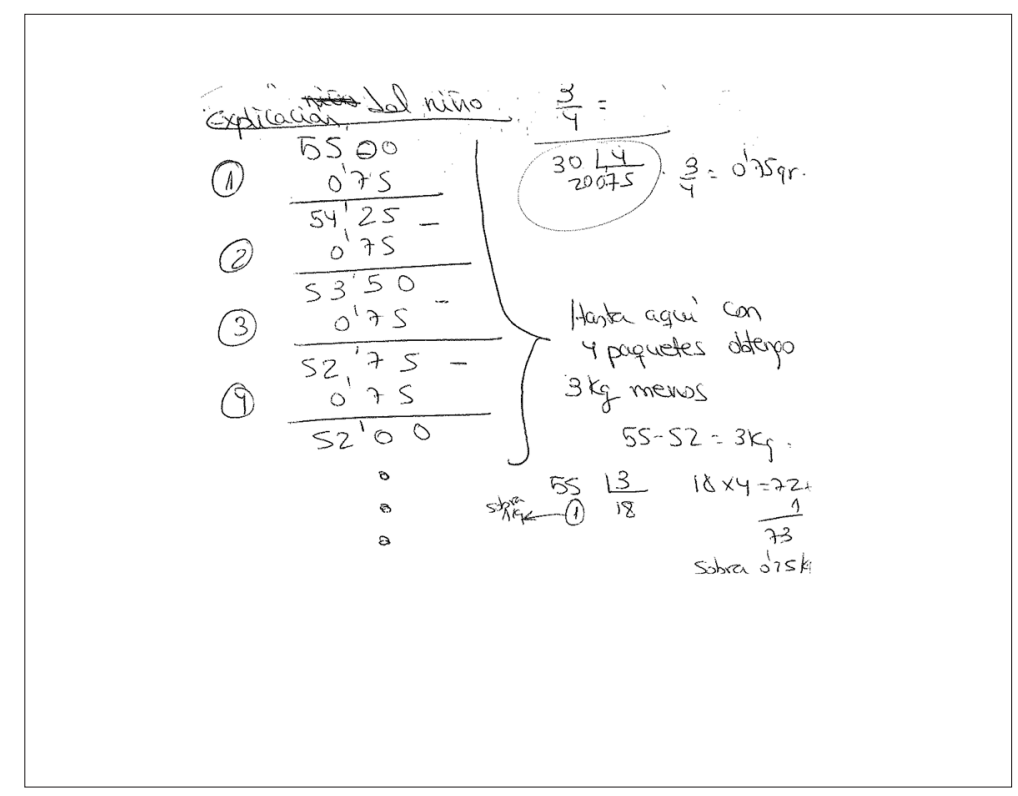

Ilustración 6. Interpretación como resta reiterada

- Conexión auxiliar: Relación entre las propiedades de la suma y multiplicación de los números racionales con la resolución de ecuaciones.

Dos EPM han planteado la siguiente ecuación:

$$
55=x \frac{3}{4}
$$

En ella, la que la incógnita es el número de paquetes, aplicando correctamente las propiedades referidas al inverso de un número respecto de la multiplicación.

4.3. Conocimiento de la práctica matemática (KPM)

- $\quad$ Formas de proceder: Heurísticos aplicables a la resolución de problemas

Comprensión: Organizar la información

Como ya hemos comentado anteriormente al describir el indicador Representación gráfica de fracciones correspondiente a Registros, mayoritariamente no han utilizado 
gráficos, algunos de ellos lo han hecho pero sin utilidad para la resolución del problema, y unos pocos han usado una representación gráfica apropiada. Así, queremos suponer que los EPM que no han usado un diagrama para organizar la información, se han planteado un esquema mental de la situación propuesta.

\section{Comprensión: Expresar en otros términos}

Algunos de los EPM que han utilizado gráficos para organizar la información han usado esta estrategia también para expresar de otra forma el enunciado del problema. Otros, han reinterpretado el enunciado utilizando lenguaje algebraico (ver ilustración 3) y el resto lo ha hecho de forma analítica.

\section{Planificación y exploración}

Treinta y cuatro EPM plantean un atisbo de planificación para la resolución antes de entrar en los cálculos, que se queda en el inicio: "leo el problema, dibujo el problema, saco datos del problema...”.

\section{Ejecución}

En general, todos registran los cálculos de manera ordenada, aunque la mayoría no justifica los pasos dados.

\section{Verificación:}

Ninguno justifica el resultado, así como tampoco hay EPM que busquen más de un procedimiento para llegar a la solución. Sin embargo, casi todos han intentado darle sentido a la solución decimal obtenida.

\section{Conclusiones}

El análisis de las resoluciones de los EPM al problema propuesto muestra las debilidades conceptuales y procedimentales de los mismos en la división de entero por fracción. Desde desconocer la interpretación correcta de los resultados obtenidos al emplear el algoritmo de la división, hasta no saber aplicarlo, pasando por los estudiantes que ni si quiera han sido capaces de averiguar qué hacer para resolver la cuestión propuesta.

Sin embargo, también hemos encontrado alumnos que han dado interpretaciones diferentes a las esperadas, pero correctas, como la resta reiterada para formar nuevos paquetes, la resolución algebraica, reagrupamientos... nuevas representaciones que 
podrían mostrar la posible especialización de su conocimiento al proponer otra fenomenología, conexión entre diferentes ideas y el uso de un abanico de heurísticos (conocimiento de los temas, de la estructura y de la práctica).

Estos resultados corroboran los obtenidos en otros estudios (Contreras, 2013; Flores, Turner y Bachea, 2005; Gregg y Gregg, 2007; Lo y Luo, 20I2; Ma, 1999; Márquez, 2013; Nillas, 2003; Peck y Wood, 2008; Perlwitz, 2004, 2005) y ponen de relieve las deficiencias en el conocimiento matemático de los EPM referido a la división entre fracción. Esto nos debería permitir conocer qué y cómo trabajar la división entre fracción en educación obligatoria $y$, posteriormente, su contextualización o representación en la formación de maestros de primaria. Por otro lado, las autoridades educativas deberían asegurar que los estudiantes que acceden a la formación universitaria tuvieran un conocimiento matemático sólido que permitiera abordar la especialización para poder construir, posteriormente, las componentes del Conocimiento Didáctico del Contenido que completarían su especialización.

Como futura investigación, partiendo de los resultados descritos en este trabajo, cabría plantearse una nueva pregunta: apoyándonos en el mismo modelo MTSK, icómo pueden afectar estas deficiencias al desarrollo de las demás componentes del conocimiento especializado del profesor de matemáticas?, es decir, cómo será el conocimiento didáctico del contenido de un maestro que no tiene una formación adecuada en su conocimiento matemático.

\section{Referencias}

Ball, D. L., Thames, M. H. y Phelps, G. (2008). Content knowledge for teaching: What makes it special? Journal of Teacher Education, 59 (5), 399-406.

Carrillo, J. (1996). Modos de resolver problemas y concepciones sobre la matemática y su enseñanza de profesores de matemáticas de alumnos de más de 14 años. Algunas aportaciones a la metodología de la investigación y estudio de posibles relaciones. Universidad de Sevilla. Sevilla: Tesis doctoral.

Carrillo, J., Climent, N., Contreras, L .C. y Muñoz-Catalán, M. C. (20/3). Determining specialised knowledge for mathematics teaching. En B. Ubuz, C. Haser y M. A. Mariotti (Eds.). Proceedings of the CERME 8 (pp. 2985-2994). Middle East Technical University, Ankara, Turquía: ERME. Disponible en: http://www.cerme8. metu.edu.tr/wgpapers/WGI7/WgI7_Climent.pdf. 
Conner, A. M., Wilson, P. S. y Jung Kim, H. (20II). Building on mathematics events in the classroom. ZDM Mathematics Education, 43, 979-992.

Contreras, M. (20I3). Problemas multiplicativos relacionados con la división de fracciones. Un estudio sobre su enseñanza y aprendizaje. Universidad de Valencia. Tesis doctoral. Disponible en: http://roderic.uv.es/handle/I0550/27397

Echenique, I. (2006). Matemáticas. Resolución de problemas. Departamento de Educación. Gobierno de Navarra.

Fennema, E. y Franke, L. M. (1992). Teachers' knowledge and its impact. In D. A. Grouws (Ed.), Handbook of research on mathematics teaching and learning (pp. 147164). New York, NY: Macmillan.

Flores, A., Turner, E. y Bachean, R. C. (2005). Posing problems to develop conceptual understanding: two teachers make sense of division of fractions. Teaching Children Mathematics, I2 (3), II7-I2I.

Flores, A. (2002). Profound understanding of division of fractions. In B. Litwiller y G. Bright, (Eds.), Making sense of fractions, ratios and proportions. (pp. 237-246). Reston, Virginia: National Council of Teachers of Mathematics.

Flores, E., Escudero, D. I. y Carrillo, J. (2013). A theoretical review of specialised content knowledge. En B. Ubuz, C. Haser y M. A. Mariotti (Eds.). Proceedings of the CERME 8 (pp. 2055-3064). Middle East Technical University, Ankara, Turquía: ERME. Disponible en: http://cerme8.metu.edu.tr/wgpapers/ WGI7/WGI7_Escudero.pdf

Godino, J. D. (2009). Categorías de análisis de los conocimientos del profesor de matemáticas. UNIÓN Revista Iberoamericana de Educación Matemática, 20, I3-3I.

Gómez, B. (2013). Formas textuales en la división. En L. Rico, M. C. Cañadas, J. Gutiérrez, M. Molina e I. Segovia (eds.), Investigación en Didáctica de la Matemática. Homenaje a Encarnación Castro. (pp. 5 I-58). Granada, España: Editorial Comares.

Gregg, J. y Gregg, D. U. (2007). Measurement and Fair-Sharing Models for Dividing Fractions. Mathematics Teaching in the Middle School, 12 (9), 490-496.

Kantowski, M. G. (198I). Problem Solving. En E. Fennema (Ed.), Mathematics Education Research: Implications for the 80'. Reston, VA: NCTM.

Liñán, M. M.y Contreras, L. C. (20I3). Debilidades y Fortalezas en el Conocimiento de los Temas en Geometría de los Estudiantes para Maestro. En A. Berciano, 
G. Gutiérrez, A. Estepa y N. Climent (Eds.), XVII Simposio Investigación en Educación Matemática. Bilbao, España: Sociedad Española de Investigación Matemática SEIEM.

Lo, J .J. y Luo, F. (20I2). Prospective elementary teachers' knowledge of fraction division. Journal of Mathematics Teacher Education, I5 (6), 48I-500.

Ma, L. (1999). Knowing and teaching elementary mathematics: teachers' understanding of fundamental mathematics in China and the United States. Mahwah, NJ: Lawence Erlbaum.

Márquez, A. I. (20I3). Conocimiento profesional de un grupo de profesores sobre la división de fracciones. Trabajo de investigación tutelada. Departamento de Didáctica de la Matemática. Universidad de Granada. Granada. Disponible en: http://fqm 193. ugr.es/media/grupos/FQM193/cms/Ana_Márquez.pdf.

Montes, M., Aguilar, A., Carrillo, J. y Muñoz-Catalán, M. C. (2013). MTSK: from Common and Horizon Knowledge to Knowledge of Topics and Structures. En B. Ubuz, C. Haser y M. A. Mariotti, Proceedings of the CERME 8 (pp. 3185-3194), Middle East Technical University, Ankara, Turquía: ERME. Disponible en: http:// cerme8.metu.edu.tr/wgpapers/WG I7/WGI7_Montes.pdf

Muñoz-Catalán, M. C. (20I2). El desarrollo profesional de una maestra novel. Un estudio de caso en un entorno colaborativo centrado en la enseñanza de las Matemáticas (Tomos I-V). Saarbrücken (Alemania): LAMBERT Academic Publishing GmbH \& Co. KG.

National Council of Teacher of Mathematics (2000). Principles and Standards for School Mathematics. Reston, VA: NCTM.

Nillas, L. (2003). Division of Fractions: Preservice Teachers' Understanding and Use of Problem Solving Strategies. The Mathematics Educator, 7 (2), 96-I I 3.

Peck, S. y Wood, J. (2008). Elastic, Cottage Cheese, and Gasoline: Visualizing Division of Fractions. Mathematics Teaching in the Middle School, I4 (4), 208-2I 2.

Perlwitz, M. D. (2004). Two students' constructed strategies to divide fractions. Mathematics Teaching in the Middle School, 10 (3), I22-126.

Perlwitz, M. D. (2005). Dividing fractions: Reconciling Self-generated solutions with algorithmic answers. Mathematics Teaching in the Middle School, I0 (6), 278-283.

Petrou, M. y Goulding, M. (20II). Conceptualising Teachers' Mathematical Knowledge in Teaching. En T. Rowland y K. Ruthven (Eds.), Mathematical Knowledge in Teaching (pp. 9-26). Melbourne: Springer. 
Polya, G. (1995). Cómo plantear y resolver problemas. México: Trillas.

Real Decreto 15/3/2006, de 7 de diciembre, por el que se establecen las enseñanzas mínimas de la Educación Primaria. Boletín Oficial del Estado, 8 de diciembre de 2006, núm. 293, 43053-43102.

Schoenfeld, A. H. (Ed.) (1994). Mathematical thinking and problem solving. Hillsdale, NJ: Lawrence Erlbaum Associates, Inc.

Shulman, L. S. (1986). Those who understand: Knowledge growth in teaching. Educational Researcher, I5 (2), 4-I4.

\section{Notas}

I. MTSK Mathematics Teacher's Specialized Knowledge

2. Problema Aritmético Elemental

3. Mathematical Proficiency for Teaching

4. Ver la descripción en el apartado Resolución de Problemas.

5. Obviaremos la fase 0 referida por Carrillo (op. cit.), puesto que hace referencia a cuestiones actitudinales que no han sido observadas en este estudio. Asimismo, mencionaremos en cada fase solamente aquellos heurísticos que consideramos pueden ser utilizados en la resolución de nuestro problema.

6. Adscrito a la Universidad de Sevilla 